\title{
Temperature Dependence of the Biotin-Avidin Bond-Rupture Force Studied by Atomic Force Microscopy
}

\author{
Yu-Shiu Lo, ${ }^{\dagger}$ Jack Simons, ${ }^{\ddagger}$ and Thomas P. Beebe, Jr.*,\$ \\ Department of Chemistry, University of Utah, Salt Lake City, Utah 84112, and Department of Chemistry and \\ Biochemistry, University of Delaware, Newark, Delaware 19716
}

Received: April 2, 2002

\begin{abstract}
We report for the first time an atomic force microscopy (AFM) investigation of the dependence of unbinding force on temperature at controlled loading rate. AFM force measurements of biotin-avidin interactions were acquired at various temperatures ranging from 13 to $37^{\circ} \mathrm{C}$ using force loading rates that are slow enough to ignore dissipative friction and to assume thermal equilibrium. With our established Poisson statistical analysis method, the unbinding force at a fixed loading rate between an individual biotin-avidin pair was extracted and was found to decrease by $\sim 5$-fold with increasing temperature over this range. On the basis of a thermodynamic model similar to that of Bell, but developed here with additional insights, the bond stiffness, effective unbinding length, and the critical unbinding energy per biotin-avidin complex were estimated. This work takes quantitative AFM studies to the next level by allowing energies to be determined from force measurements.
\end{abstract}

\section{Introduction}

Forces between biotin-avidin pairs are dynamic quantities that undergo fluctuations as thermal motions cause the pairs to undergo movements in their interpair distances $r$. Near the equilibrium distance $r_{\mathrm{e}}^{0}$, these forces are small. As $r$ increases, a restoring force develops and grows until it reaches a maximum at $r_{\text {crit }}$, after which it decreases until the linkage eventually is fully dissociated. Such behavior is characteristic of all chemical bonds, and the intermolecular potential that varies with $r$ is depicted in Figure 1. When an outward bias force $F_{\text {out }}$ is applied to such a linkage, the net restoring force (i.e., inward toward smaller $r$ ) experienced by the pair is $-F_{\text {out }}+F_{\text {restoring, where }}$ the latter is due to the bonding and other attractive forces that link the biotin ligand to the avidin receptor in a manner characterized by the potential of Figure 1. For $r>r_{\mathrm{e}}^{0}$ but near this minimum, this net force is outward so the ligand-receptor linkage becomes stretched. As $r$ is increased, the net force becomes less outward (because $F_{\text {restoring grows) until the restoring }}$ and bias forces reach a balance. However, there exists a critical distance $r_{\text {crit }}$ where $F_{\text {restoring }}$ is largest; beyond $r_{\text {crit }}$, the restoring force decreases and eventually reaches zero at very large $r$. For bias forces that exceed the restoring force at $r_{\text {crit }}$, the linkage will stretch beyond $r_{\text {crit }}$ until the linkage is fully ruptured. It is this maximum restoring force that we measure and deal with in this paper.

The above characteristics of ligand-receptor interactions cause the minimum bias force needed to effect unbinding to be somewhat ill-defined. For any finite bias force, the net restoring force will eventually reach zero once enough thermal energy has been deposited into the linkage to move $r$ to a position where $F_{\text {restoring }}$ is less than the bias force. For very small bias forces,

* Corresponding author. E-mail: beebe@udel.edu. Phone: (302) 8311888. Fax: (302) 831-6335.

$\dagger$ Present address: 12 F. No. 307, Kuang-Fu Rd. Sec. 2, Hsinchu 300, Taiwan.

$\doteqdot$ University of Utah

$\S$ University of Delaware.

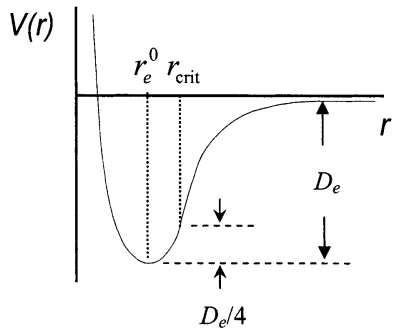

Figure 1. Intermolecular potential within the ligand-receptor pair. The interaction is modeled with a Morse potential, whereas near the potential minimum $\left(r_{\mathrm{e}}^{0}\right)$ it is approximated with a harmonic potential for simplicity. Beyond the point $r_{\text {crit }}$ (where $|\mathrm{d} V / \mathrm{d} r|$ is largest), the restoring force acting on the biotin-avidin complex decreases. If the external force exceeds this $|\mathrm{d} V / \mathrm{d} r|$, the bond restoring force can no longer dominate the pulling force exerted by the AFM cantilever, and so rupture occurs.

this will require waiting until thermal motions have caused the linkage to move far beyond $r_{\text {crit. }}$. However, if the applied bias force exceeds the maximum restoring force (i.e., $F_{\text {restoring }}$ at $r_{\text {crit }}$ ), the linkage will promptly rupture. In most of the experiments that we discuss in this paper, the bias force is "ramped" upward from zero until the linkage is observed to rupture. The rate at which this force undergoes such ramping can vary substantially from laboratory to laboratory and needs to be carefully considered when interpreting the data, as we illustrate later in this paper.

The loading-rate dependence of ligand-receptor unbinding forces (so-called "dynamic force spectroscopy") has been studied experimentally ${ }^{1-6}$ and theoretically ${ }^{7-10}$ in several different laboratories, including our own. In our experiments, we argue below that the range of loading rates used here allowed us to determine unbinding forces (i.e., the force at which the AFM tip breaks free from the surface) which (1) are not simply artifacts obtained by waiting long enough for thermal energy to break the linkage and (2) are related directly to the ligandreceptor attractive potential energy (in particular to the energy required to access the critical distance $r_{\text {crit }}$ discussed earlier). 
Temperature is another important parameter that affects the rate of ligand-receptor dissociation in both the absence and presence of applied bias forces. At higher temperatures, one has to wait less time for thermal excitation to induce linkage rupture under any applied bias force. Here, for the first time, an AFM investigation of the temperature dependence of unbinding forces is presented. A thermodynamic-kinetic model similar to that used by Bell ${ }^{11}$ is established and further developed to help interpret the temperature dependence of the biotinavidin bond-rupture forces and to extract important thermodynamic parameters.

In the AFM experiments discussed in the following section, the relationship between the force needed to effect tip-substrate breakaway in any particular measurement and the potential energy that binds a biotin ligand to an avidin binding site is complicated in two independent ways.

First, each time the AFM tip touches the substrate, it is possible for various numbers $(n)$ of biotin-avidin linkages to develop. Thus, each time the tip is withdrawn from the substrate until the force reaches a point at which the tip breaks free, one may be probing a different number of linkages. However, as we discuss later, and as has been proven in our past work in this area, ${ }^{13-18}$ the probability, $P(n)$, that a given measurement has $n$ biotin-avidin linkages holding the tip to the substrate follows a Poisson distribution. Hence, by determining the breakaway force for a large number of such measurements, and using the Poisson distribution, one can extract the breakaway force for the $n=1$ case (i.e., for a single biotin-avidin linkage).

The second complication involves the kinds of forces (e.g., hydrogen bonds, van der Waals interactions, polar group attractions, etc.) that are operative within each biotin-avidin linkage and at what stage in the fragmentation these forces act. As shown beautifully by Izraelev et al. in ref 10, especially on pp 1571-1572, each such linkage can involve a variety of attractive forces. Moreover, the biotin can be bound to various sites within the avidin "pocket" (see Figures 5, 7, and 8 in ref 10) at various times. As a result, when an individual biotin is withdrawn (via an applied bias force) from an avidin pocket, one expects to observe a series of plateaus in the location of the biotin as a function of time. These plateaus should occur as the force applied to the biotin is ramped up, causing the biotin to move from one binding site to another within the pocket. Indeed, such behavior is observed in molecular dynamics simulations of such single biotin-avidin pullaway experiments. For example, one sees this clearly in Figure 5 of ref 10, where it is shown that the maximum force corresponds to breaking not the last but the strongest linkage of biotin within the pocket.

The above observations suggest the following:

1. By assuming a Poisson distribution of the number of biotin-avidin linkages present in each measurement, we can infer a maximum force $F_{i}$ characteristic of a single such linkage.

2. The force needed to effect rupture of even a single linkage measures not any single hydrogen bond or ionic interaction. Rather, it more likely relates to the rupture of several such attractive interactions that is needed to remove biotin from its strongest binding site within an avidin pocket (as illustrated in Figure 5 of ref 10).

\section{Experimental Section}

To conduct AFM force measurements at various temperatures, we adjusted the temperature of the entire AFM apparatus between 13 and $37{ }^{\circ} \mathrm{C}$. For measurements taken below $20^{\circ} \mathrm{C}$ or above $30{ }^{\circ} \mathrm{C}$, ice packs or heating tapes were applied to a thermal shroud surrounding the instrument to cool or heat the
(A)

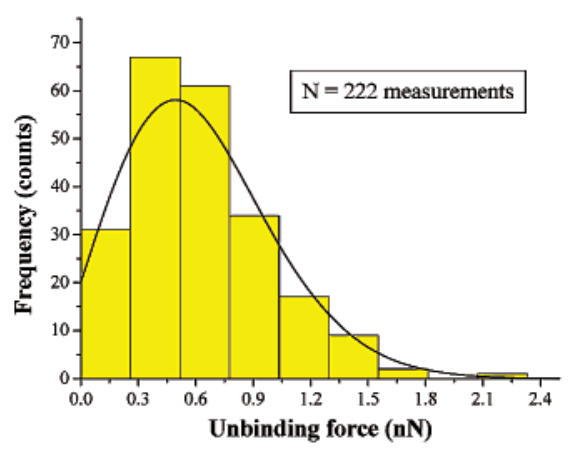

(B)

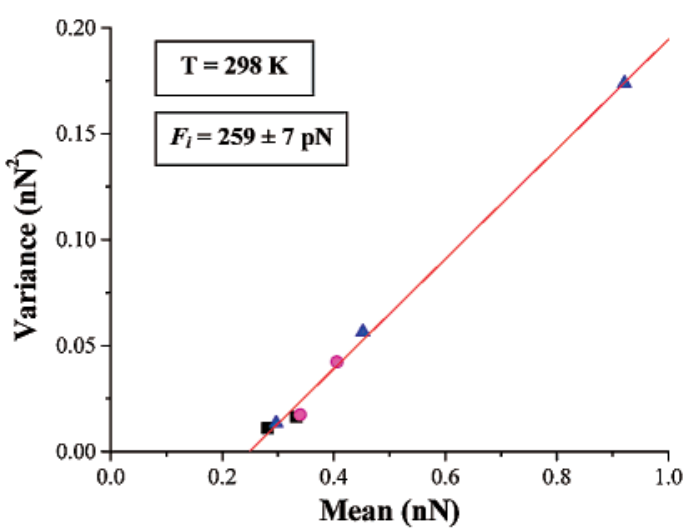

Figure 2. Application of the Poisson analysis method to AFM force measurements. (A) Histogram of the unbinding forces from one isothermal combined set of 222 measurements between an avidinmodified AFM tip and a biotinylated bovine serum albumin (BBSA)modified substrate under $\mathrm{pH} 7$ phosphate-buffered saline (PBS). The data are fitted well with a Poisson distribution curve. (B) Variance vs mean plot of the BBSA-avidin system. Each data point represents a set of multiple measurements using a particular tip at a particular area. Different symbols are for different tips modified by the same chemistry. Based on the Poisson analysis (see Appendix), the slope of the linear regression curve gives the magnitude of individual biotin-avidin unbinding force $F_{\mathrm{i}}$. It was determined to be $259 \pm 7 \mathrm{pN}$ for the measurements made at $T=298 \mathrm{~K}$ and a loading rate of $2.0 \times 10^{5}$ $\mathrm{pN} \cdot \mathrm{s}^{-1}$.

AFM stage sitting inside a box surrounded by foam. The temperature fluctuations during a given set of measurements, as measured by a chromel-alumel thermocouple situated near the sample stage, were within $\pm 1{ }^{\circ} \mathrm{C}$ of the set-point temperature, and extensive control experiments were made to determine drift rates that were used to set measurement time scales appropriately. ${ }^{12}$ The biotin- and avidin-modified AFM tips and substrates were prepared using a protocol described in detail previously, ${ }^{13}$ and extensive surface analytical methods were used to confirm the putative tip and surface modification procedures. These analyses by XPS and TOF-SIMS, though not the focus of the present paper, were described in detail in previous publications. ${ }^{13,16,17}$

To extract the unbinding forces between individual biotinavidin pairs, we used an established statistical analysis method based on the properties of the Poisson distribution that our earlier work $^{13-18}$ and that of others ${ }^{19-21}$ has shown to be reliable (see Appendix). The application of the Poisson analysis method is demonstrated in Figure 2A, where data pertinent to the present study are shown to fit well the assumed Poisson functional form. We should note that the range of forces caused by the variation in the number $(n)$ of biotin-avidin linkages present is ca. $2 \mathrm{nN}$ (see Figure 2A), which is even larger than the ca. $100-400 \mathrm{pN}$ range in single-linkage forces (see Figure $2 \mathrm{~B}$ ) experienced as the temperature is varied (see Figure 3). However, because the 


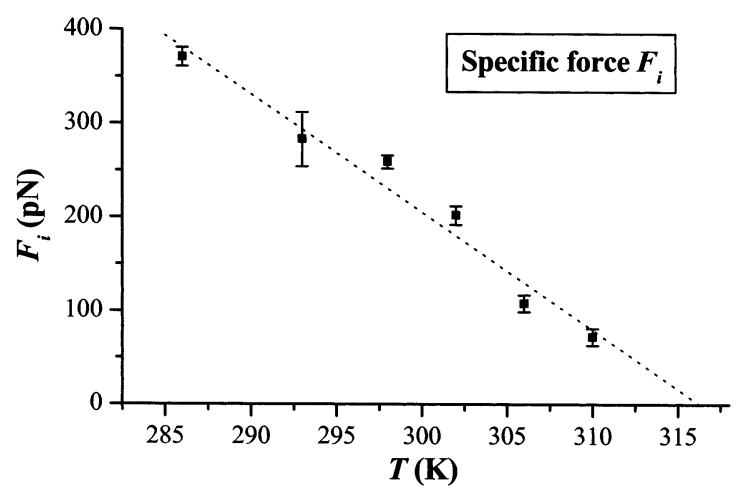

Figure 3. Temperature dependence of individual biotin-avidin unbinding forces $F_{\mathrm{i}}$. The values of $F_{\mathrm{i}}$ are determined by the Poisson method from the variance vs mean plots (as shown in Figure 2B). The error bars correspond to one standard deviation. $F_{\mathrm{i}}$ has a significant dependence on temperature with a slope of $-12.6 \pm 1.2 \mathrm{pN} \cdot \mathrm{K}^{-1}$

forces obtained for $n$ linkages follow the Poisson distribution very well, we are able to extract single-linkage forces $F_{i}$ with precisions of better than $50 \mathrm{pN}$ in most cases (see the error bars in Figure 3). This then allows us to extract from the temperature variation in such single-linkage forces the desired single-linkage energetics, as discussed in the following section.

\section{Results and Discussion}

For the reasons discussed in the Introduction, single-linkage bond-rupture forces depend on temperature and on the rate of force loading. In this study, all force-distance measurements were performed with cantilevers of the same nominal force constant $\left(0.039 \pm 0.004 \mathrm{~N} \cdot \mathrm{m}^{-1}\right.$; each cantilever was individually calibrated $)^{22}$ at a tip scan speed of $5 \mu \mathrm{m} \cdot \mathrm{s}^{-1}$, which corresponded to a nominal loading rate of $(2.0 \pm 0.2) \times 10^{5} \mathrm{pN} \cdot \mathrm{s}^{-1}$. In contrast, the dynamics simulations discussed in ref 10 have loading rates of ca. $10^{12} \mathrm{pN} \mathrm{s}^{-1}$, which are orders of magnitude higher than those used here. As discussed below, the fact that our loading rate is so slow allows us to neglect dissipative frictions that cannot be ignored in the dynamics simulations of ref 10. Moreover, we can assume that the biotin-avidin pairs remain in thermal equilibrium throughout the tip-pulling process because of the slow loading rate.

For our conditions, the rupture time of the AFM force measurement $\tau_{\mathrm{R}}$ was estimated to be on the millisecond scale. ${ }^{23}$ The individual biotin-avidin unbinding forces $\left(F_{i}\right)$ at six different temperatures over the range of 13 to $37{ }^{\circ} \mathrm{C}$ were determined with the Poisson method and are plotted in Figure 3 . We again note that the distribution of forces obtained in the 200-plus individual measurements at each temperature fit the expected Poisson distribution well enough that the resultant error bars in the single-linkage forces $F_{i}$ are small, as indicated in Figure 3.

The binding of streptavidin and avidin with biotin is one of the strongest noncovalent ligand-receptor interactions known $\left(K_{\mathrm{a}} \approx 10^{14}-10^{15} \mathrm{M}^{-1}\right) .{ }^{24}$ As discussed earlier, each individual biotin-avidin or biotin-streptavidin linkage is comprised of a multiple hydrogen bond, polar group, and van der Waals interactions. ${ }^{24-27}$ To interpret the temperature dependence of unbinding forces observed in our AFM experiments, we employ a thermodynamic approach that focuses on how the applied force reduces the amount of thermal energy ${ }^{28}$ that must be obtained from the surroundings. For simplicity, we assume that the model of biotin-avidin binding offered in ref 10 is essentially correct. In particular, we assume that the applied bias force causes the biotin to move from one binding site to another within the avidin

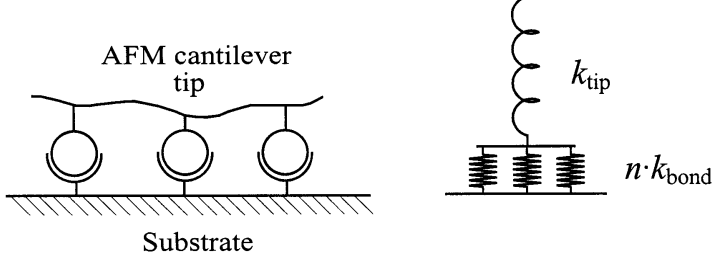

Figure 4. Schematic of binding between ligands immobilized on an AFM tip and receptors immobilized on a substrate. The $n$ ligandreceptor pairs are depicted as separate springs with a total force constant of $n k_{\text {bond. }}$. The AFM tip is attached to a soft cantilever that is treated as a spring with a force constant of $k_{\text {tip }} \ll n k_{\text {bond }}$ for reasons that are discussed and justified in the text $\left(n k_{\text {bond }} / k_{\text {tip }} \approx 450\right)$.

pocket. As the tip force is ramped up, the force causes the biotin to so move until it reaches the site with the strongest binding. Once the bias force causes the biotin to break free from this strongest site, the biotin can fully exit the pocket as it moves even further under the bias force. Thus, the individual biotinavidin unbinding forces $F_{i}$ extracted from our data are assumed to relate to this strongest site's binding.

We next model the biotin-avidin binding potential for this strongest site within the pocket in terms of a potential function of the Morse form. Our use of the Morse form for the biotinavidin strong-site potential is not meant to reflect any assumptions we make about the nature of the binding at this site. Moreover, the few $(n)$ biotin-avidin complexes that remain bound immediately prior to the pull-off point are assumed to be arranged in a manner that causes all of them to be similarly loaded as the tip is retracted from the substrate. ${ }^{29}$ Our past work has shown that, should this assumption not hold, a clear pattern of curvature in the variance vs mean force plots (e.g., see Figure 2B) would be evident. Such curvature was not observed in the present study.

We realize that each site in the pocket involves hydrogen bonds, polar group attractions, and van der Waals interactions. However, all such potentials share the characteristics of the potential displayed in Figure 1. We choose to describe all such potentials in the Morse form because, for such a function, we can straightforwardly determine the critical distance $r_{\text {crit }}$ (see the introduction) as well as the energy at this $r, V\left(r_{\text {crit }}\right)$ in terms of the basic parameters of the Morse model. Other functional forms can be employed using our general methodology.

Let us now imagine removing a tip from the substrate under conditions where the tip contains $n$ biotin-avidin linkages as graphically depicted in Figure 4 . Bending the cantilever by a length $d$ exerts a force $k_{\text {tip }} d$ on the complex, where $k_{\text {tip }}$ is the cantilever force constant. This stretching force is opposed by the $n$ biotin-avidin complexes' internal bonding forces, which balance the applied force when the biotin-avidin distances are stretched by an amount $\delta r$ in the direction of the AFM cantilever tip. As a result, the opposing forces reach a balance and the biotin-avidin distances move to new equilibrium positions where $n k_{\text {bond }} \delta r=k_{\text {tip }}(d-\delta r)$. Here $k_{\text {bond }}$ is an individual complex's force constant $k_{\text {bond }}=\partial^{2} V / \partial r^{2}$, and $n$ is the number of biotin-avidin complexes present. This result allows us to express the lengthening of the distances as $\delta r=k_{\text {tip }} d /\left(k_{\text {tip }}+\right.$ $\left.n k_{\text {bond }}\right)$ and the amount of energy deposited as potential energy within each of the $n$ linkages as $1 / 2 k_{\text {bond }}\left(k_{\text {tip }} d /\left(k_{\text {tip }}+n k_{\text {bond }}\right)\right)^{2}$. For slow rates of pulling and within the millisecond time scale of our AFM experiment, we assume that the system is at "pseudo-equilibrium" at any moment along the unbinding process. We note that this situation differs qualitatively from what happens in the molecular dynamics simulations of ref 10 where the force loading rate was orders of magnitude larger. 
Such extremely large rates were required in the latter case to allow the simulations to achieve biotin-avidin rupture within a time duration that could be accessed in the simulation. In our experiments, the tip moves slowly enough that it is safe to assume that each biotin-avidin complex is in thermal equilibrium with its surroundings. This assumption allows us to use the equilibrium Maxwell-Boltzmann energy distribution formula to compute the probability of various amounts of thermal energy being available to the each biotin-avidin complex.

During the unbinding process in the AFM measurement, the energy that must be deposited into the $n$ biotin-avidin complexes in the form of thermal energy to effect rupture of the $n$ linkages is assumed to have terms that account for

(1) $n$ times the amount $\Delta E^{\ddagger}$ needed to effect rupture of a single linkage in the absence of an external force $\left(\Delta E^{\ddagger}\right.$ is not assumed to equal the full dissociation energy of a biotin-avidin complex but, as detailed later, is only a fraction of the energy needed to dislodge biotin from avidin's strongest binding site),

(2) minus a term for the mechanical energy deposited into the $n$ biotin-avidin linkages by the AFM cantilever,

(3) plus a term for the energy dissipated due to viscous drag from operation at a finite rate of pulling $v$ (this energy can, as shown below, be ignored because of the slow loading rate).

As a result, the net critical energy barrier $\delta E_{\mathrm{T}}$ to be surmounted by thermal excitation before rupture of the $n$ linkages occurs is

$$
\delta E_{\mathrm{T}}=n \Delta E^{\ddagger}-\frac{1}{2} n k_{\mathrm{bond}}\left(\frac{k_{\mathrm{tip}}}{k_{\mathrm{tip}}+n k_{\mathrm{bond}}}\right)^{2} d^{2}+\frac{\gamma v d k_{\mathrm{tip}} n}{k_{\mathrm{tip}}+n k_{\mathrm{bond}}}
$$

The characteristic time needed to rupture the $n$ bonds $\tau_{\mathrm{R}}$ can be related to the time $\tau_{\mathrm{D}}$ needed for biotin to move out of avidin's binding pocket in the absence of any bias force or of any attractive forces as

$$
\tau_{\mathrm{R}}=\tau_{\mathrm{D}} \mathrm{e}^{\delta E_{\mathrm{T}} / n k_{\mathrm{B}} T}
$$

where $n k_{\mathrm{B}} T$ is the average thermal energy available in the $n$ linkages. In our theoretical treatments, $\tau_{\mathrm{D}}$ is assumed to have a negligible $T$ dependence over the narrow temperature range applied in this study. Combining with eq 1 and taking the logarithm on both sides of eq 2 and rearranging, we obtain

$$
\begin{array}{r}
n k_{\mathrm{B}} T \ln \left(\frac{\tau_{\mathrm{R}}}{\tau_{\mathrm{D}}}\right)=n \Delta E^{\ddagger}-\frac{1}{2} n k_{\mathrm{bond}}\left(\frac{k_{\mathrm{tip}} d}{k_{\text {tip }}+n k_{\text {bond }}}\right)^{2}+ \\
n v \gamma\left(\frac{k_{\text {tip }} d}{k_{\text {tip }}+n k_{\text {bond }}}\right)
\end{array}
$$

Next, we consider the case in which the bond force constant $k_{\text {bond }}$ is sufficiently greater than the cantilever's force constant $k_{\text {tip }}, k_{\text {bond }} \gg k_{\text {tip }}$, which is shown below to be valid for the experimental conditions employed here (by a factor of $\sim 450 \times$ ). In this case, eq 3 becomes

$$
n k_{\mathrm{B}} T \ln \left(\frac{\tau_{\mathrm{R}}}{\tau_{\mathrm{D}}}\right)=n \Delta E^{\ddagger}-\frac{1}{2} \frac{\left(k_{\mathrm{tip}} d\right)^{2}}{n k_{\mathrm{bond}}}+\left(\frac{k_{\mathrm{tip}} d}{k_{\mathrm{bond}}}\right) v \gamma
$$

For slow tip-scan speeds such as we employed, the factor $v \gamma$ appearing in the friction term ${ }^{10}$ is much smaller than the $k_{\text {tip }} d$ term, so the last term in eq 4 can be neglected compared to the others. For example, for $v=5 \mu \mathrm{m} \cdot \mathrm{s}^{-1}$ and $\gamma \sim 2 \times 10^{-8}$ $\mathrm{pN} \cdot \mathrm{s} \cdot \mathrm{nm}^{-1}, v \gamma \approx 10^{-4} \mathrm{pN}$; by comparison, typical values for the $k_{\text {tip }} d$ term are $0.039 \mathrm{~N} \cdot \mathrm{m}^{-1} \times 10^{-9} \mathrm{~m}=390 \mathrm{pN}$.
For each component of eq 4 to scale linearly with the number of linkages present $(n)$, as it must to reproduce the observed Poisson distribution of forces, the AFM tip force $k_{\text {tip }} d$ must be linearly proportional to $n$. That is, $k_{\text {tip }} d=F_{\text {tot }}=n F_{\text {i }}$, where $F_{\mathrm{i}}$ is the force needed to displace an individual biotin from avidin's strongest binding site. This means that the force needed to effect rupture within time $\tau_{\mathrm{R}}$ when $n$ linkages are present is $n$ times the force needed to rupture one linkage in this same time. Substituting this into eq 4 and dividing both sides by $n$, we obtain

$$
k_{\mathrm{B}} T \ln \left(\frac{\tau_{\mathrm{R}}}{\tau_{\mathrm{D}}}\right)=\Delta E^{\neq}-\frac{1}{2} \frac{F_{i}^{2}}{k_{\mathrm{bond}}} \gamma
$$

Hence, for slow tip scan speeds and soft cantilevers, the temperature dependence (at constant loading rate) of individual biotin-avidin rupture forces can be expressed as

$$
F_{\mathrm{i}}^{2}=2 \Delta E^{\ddagger} k_{\text {bond }}-2 k_{\mathrm{B}} T k_{\text {bond }} \ln \left(\frac{\tau_{\mathrm{R}}}{\tau_{\mathrm{D}}}\right)
$$

which is the basic working equation that we use to analyze our data. Note that it is the square of the force $\left(n F_{\mathrm{i}}\right)^{2}=\left(k_{\text {tip }} d\right)^{2}$ that enters into eq 4 because this square is proportional to energy and it is an energy balance that leads from eq 3 ultimately to eq 6 .

Further simplifications are possible. With the typical bondrupture time in our AFM experiment $\tau_{\mathrm{R}} \approx 10^{-3} \mathrm{~s}$ and using the diffusion time estimated by earlier workers $\tau_{\mathrm{D}} \approx 10^{-8} \mathrm{~s}$, the ratio of time scales appearing in the log term on the right of eq 6 can be estimated $\left(\tau_{\mathrm{R}} / \tau_{\mathrm{D}} \approx 10^{5}\right) .{ }^{30}$ Because this ratio appears in a logarithmic factor in eq 6 , variations by even 1 or 2 orders of magnitude around our best estimates would have only a limited effect on the bond stiffness and energy factors that we compute.

The stiffness of the ligand-receptor bond $k_{\text {bond }}$ and the critical unbinding energy $\Delta E^{\ddagger}$ to displace a single biotin from avidin's strongest binding site can thus be determined from the slope and the $y$-intercept of the linear regression curve of eq 6 in the $F_{\mathrm{i}}{ }^{2}$ vs $T$ plot (Figure 5) as $17.4 \pm 1.4 \mathrm{~N} \cdot \mathrm{m}^{-1}$ and $7.1 \pm 0.8$ $\mathrm{kcal} \cdot \mathrm{mol}^{-1}$, respectively. It should be kept in mind that these values relate to a single biotin-avidin linkage which, as noted earlier, contains several hydrogen bonds, polar interactions, and van der Waals attractions. As one of the strongest known noncovalent ligand-receptor interactions, the stiffness of the biotin-avidin complex obtained above appears reasonable and supports the assumption made in the derivation that $k_{\text {bond }}$ (i.e., $\left.17.4 \pm 1.4 \mathrm{~N} \cdot \mathrm{m}^{-1}\right) \gg k_{\text {tip }}\left(0.039 \pm 0.004 \mathrm{~N} \cdot \mathrm{m}^{-1}\right)$ by a factor of $\sim 450$.

Let us now discuss the meaning of the result $\Delta E^{\ddagger}=7.1 \pm$ $0.8 \mathrm{kcal} \cdot \mathrm{mol}^{-1}$. As discussed earlier, we assume that to effect bond rupture under the influence of the external force $F_{\mathrm{AFM}}=$ $k_{\text {tip }} d$, the intermolecular separation between biotin and avidin must move (by tip pulling and thermal motion combined) beyond a point $r_{\text {crit }}$ (see Figure 1) where $|\mathrm{d} V / \mathrm{d} r|$ is largest. Beyond $r_{\text {crit }}$, the restoring force acting on the biotin-avidin complex decreases so it can no longer overcome the bias force exerted by the AFM cantilever, and the rupture is inevitable. For a Morse potential

$$
V(r)=D_{\mathrm{e}}\left(1-\mathrm{e}^{-b\left(r-r_{\mathrm{e}}^{0}\right)}\right)^{2}
$$

the energy required to move along the reaction coordinate from the equilibrium position $r_{\mathrm{e}}^{0}$ to $r_{\text {crit }}$ is $\Delta E^{\ddagger}=V\left(r_{\text {crit }}\right)-V\left(r_{\mathrm{e}}^{0}\right)=$ 


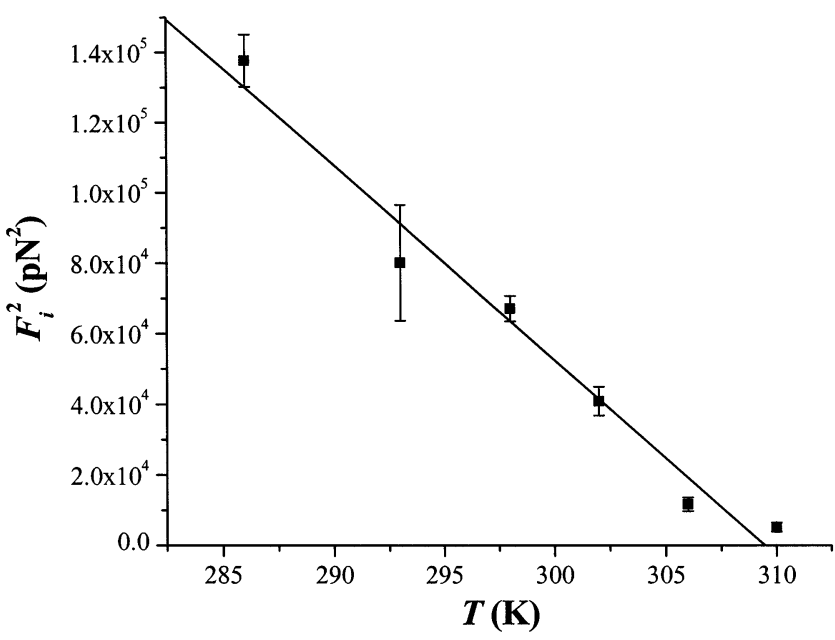

Figure 5. Plot of $F_{\mathrm{i}}^{2}$ vs $T$. Based on our theoretical derivation with a hybrid kinetic - thermodynamic approach, the temperature dependence of individual biotin-avidin unbinding force $F_{\mathrm{i}}$ can be expressed as $F_{\mathrm{i}}^{2}=2 \Delta E^{\ddagger} k_{\text {bond }}-2 k_{\mathrm{B}} T k_{\text {bond }} \ln \left(\tau_{\mathrm{R}} / \tau_{\mathrm{D}}\right)$. With the estimation of $\tau_{\mathrm{R}} / \tau_{\mathrm{D}} \approx$ $10^{5}$ (see text), the stiffness of the biotin-avidin bond $k_{\text {bond }}$ and the critical unbinding energy $\Delta E^{\ddagger}$ can be determined from the slope $\left(-5543 \pm 455 \mathrm{pN}^{2} \cdot \mathrm{K}^{-1}\right)$ and the $y$-intercept $\left((1.72 \pm 0.14) \times 10^{6} \mathrm{pN}^{2}\right)$ of the linear regression curve. $k_{\text {bond }}$ and $\Delta E^{\ddagger}$ are calculated as $17.4 \pm$ $1.4 \mathrm{~N} \cdot \mathrm{m}^{-1}$ and $7.1 \pm 0.8 \mathrm{kcal} \cdot \mathrm{mol}^{-1}$, respectively.

$D_{\mathrm{e}} / 4$, where $D_{\mathrm{e}}$ is the equilibrium dissociation energy. ${ }^{31} \mathrm{Using}$ the present determination of $\Delta E^{\ddagger}$ as $7.1 \pm 0.8 \mathrm{kcal} \cdot \mathrm{mol}^{-1}, D_{\mathrm{e}}$ is thus estimated to be $28.4 \pm 0.8 \mathrm{kcal} \cdot \mathrm{mol}^{-1}$. This value is in reasonably good agreement with independent determinations of the enthalpy change for biotin-avidin dissociation, ${ }^{32} \Delta H=23.4$ $\mathrm{kcal} \cdot \mathrm{mol}^{-1}$. A binding energy of ca. $28 \mathrm{kcal} \mathrm{mol}^{-1}$ seems to be consistent with the variety of hydrogen bond, polar group, and van der Waals attractions that seem (see ref 10 Figures 5-7) to characterize the binding sites in avidin. Moreover, the $k_{\text {bond }}$ value of $17.4 \pm 1.4 \mathrm{~N} \cdot \mathrm{m}^{-1}$ is consistent with what is expected for hydrogen bonds. ${ }^{33}$

The bond force constant is the second derivative of the potential at the equilibrium position. For a Morse potential, we obtain

$$
k_{\text {bond }}=\left.\frac{\mathrm{d}^{2} V(r)}{\mathrm{d} r^{2}}\right|_{r_{\mathrm{e}}^{0}}=2 b^{2} D_{\mathrm{e}}
$$

Inserting the values of $k_{\text {bond }}$ and $D_{\mathrm{e}}$ obtained above, the Morse potential parameter $b$ is calculated ${ }^{34}$ to be $(6.6 \pm 0.5) \times 10^{9}$ $\mathrm{m}^{-1}$. Again, for a Morse potential, one can determine $r_{\text {crit }}-r_{\mathrm{e}}^{0}$ by finding where $\mathrm{d}^{2} V / \mathrm{d} r^{2}=0$, and one obtains $\left(r_{\text {crit }}-r_{\mathrm{e}}^{0}\right)=$ $\ln 2 / b \approx 1.0 \pm 0.1 \AA$. This critical displacement magnitude is in reasonable agreement with the mapped energy barrier of biotin-avidin unbinding projected along the dissociation coordinate $(\approx 1.2 \AA)$ obtained from previous dynamic force spectroscopy studies at the loading rate applied in this work. ${ }^{2,6}$

\section{Conclusion}

This first analysis of the temperature dependence of AFM unbinding forces revealed that temperature has a significant effect on the rupture forces measured by AFM. It also shows how several thermodynamic parameters, including bond stiffness, critical linkage displacement, and critical unbinding energy, can be extracted from the temperature dependence of the unbinding force. A slow loading rate and a soft cantilever played important roles in allowing us to use a straightforward quasiequilibrium model to extract such parameters from our data.
Further temperature-dependence studies of bond-rupture forces at different loading rates and in other ligand-receptor systems including related protein mutants are desirable to achieve a more thorough understanding of the kinetics and relevant thermodynamics of such ligand-receptor unbinding processes.

Acknowledgment. This work was supported by the National Science Foundation (T.P.B., CHE-9814477 and DMR-9724307; J.S., CHE-9982420), the Alfred P. Sloan Foundation (T.P.B.), and the National Institutes of Health (T.P.B., R01-NS43928).

\section{Appendix}

Poisson Statistical Analysis. The assumptions made in this method are that the total adhesive force in an AFM pulloff event is composed of a number of discrete bonds (typically one to a few tens, depending on the tip sharpness and the size of the ligands involved) and that the distribution of the number of bonds broken at the pulloff point follows a Poisson distribution. The Poisson number distribution (as seen in Figure 2A) has been demonstrated for several systems in our prior work and that of others. As defined by the properties of the Poisson distribution, the mean $\mu_{n}$ and the variance $\sigma_{n}^{2}$ of the number of chemical bonds broken at the pulloff point in the AFM experiment are the same, i.e., $\mu_{n}=\sigma_{n}{ }^{2}$. The adhesive force $m$ measured in one force-distance curve is related to the number of bonds $n$ ruptured during a pull-off event by $m=n F_{\text {i }}$, where $F_{\mathrm{i}}$ represents the average individual bond-rupture force in the system, the value that we seek to determine. A sampling of many of these pulloff events produces a mean measured pulloff force $\mu_{m}$ and a pulloff force variance, $\sigma_{m}{ }^{2}$ (note carefully the different subscripts). On the basis of the relationship between the measured force and number of bonds ruptured, we can derive the following equations: $\mu_{m}=\mu_{n} F_{\mathrm{i}}$ and $\sigma_{m}{ }^{2}=\sigma_{n}{ }^{2} F_{\mathrm{i}}{ }^{2}$. Because $\mu_{n}=\sigma_{n}^{2}$ for a Poisson process, the magnitude of $F_{\mathrm{i}}$ can be determined as $F_{\mathrm{i}}=\sigma_{m}^{2} / \mu_{m}$. Although they may not exist to any significant level in any particular system, nonspecific, longrange interactions, $F_{0}$, can be taken into account; the mean and the variance of the pulloff forces then become $\mu_{m}=\mu_{n} F_{\mathrm{i}}+F_{0}$ and $\sigma_{m}{ }^{2}=\mu_{m} F_{\mathrm{i}}-F_{\mathrm{i}} F_{0}$, respectively. A linear regression curve of the variance $\sigma_{m}{ }^{2}$ versus the mean $\mu_{m}$ of the pulloff force from several sets of measurements will give the magnitude of the individual bond-rupture force $F_{\mathrm{i}}$ and the product $-F_{\mathrm{i}} F_{0}$, as the slope and $y$-intercept, respectively. The latter quantity can be used to estimate any nonspecific "background" forces, if they are present. For reasons of space, it is not our intention to establish and validate the Poisson analysis method here. These methods have been employed in many different biological and chemical systems in our prior work ${ }^{13-17}$ and that of others. ${ }^{19-21}$

\section{References and Notes}

(1) Fritz, J.; Katopodis, A. G.; Kolbinger, F.; Anselmetti, D. Proc. Natl. Acad. Sci. U.S.A. 1998, 95, 12283-12288.

(2) Merkel, R.; Nassoy, P.; Leung, A.; Ritchie, K.; Evans, E. Nature 1999, 397, 50-53.

(3) Strunz, T.; Oroszlan, K.; Schäfer, R.; Güntherodt, H.-J. Proc. Natl. Acad. Sci. U.S.A. 1999, 96, 11277-11282.

(4) Schwesinger, F.; Ros, R.; Strunz, T.; Anselmetti, D.; Güntherodt, H.-J.; Honegger, A.; Jermutus, L.; Tiefenauer, L.; Plückthun, A. Proc. Natl. Acad. Sci. U.S.A. 2000, 97, 9972-9977.

(5) Gergely, C.; Voegel, J.-C.; Schaaf, P.; Senger, B.; Maaloum, M.; Horber, J. K. H.; Hemmer. Proc. Natl. Acad. Sci. U.S.A. 2000, 97, 1080210807. 3748 .

(6) Lo, Y.-S.; Zhu, Y.; Beebe, T. P., Jr. Langmuir 2001, 17, 3741-

(7) Grubmüller, H.; Heymann, B.; Tavan, P. Science 1996, 271, $997-$ 999.

(8) Evans, E.; Ritchie, K. Biophys. J. 1997, 72, 1541-1555. 
(9) Strunz, T.; Oroszlan, K.; Schumakovitch, I.; Güntherodt, H.-J.; Hegner, M. Biophys. J. 2000, 79, 1206-1212.

(10) Izrailev, S.; Stepaniants, S.; Balsera, M.; Oono, Y.; Schulten, K. Biophys. J. 1997, 72, 1568-1581.

(11) Bell, G. I. Science 1978, 200, 618-627.

(12) As a part of the "background" experiments in this study, we performed extensive tests of thermal drift rates and time scales of the measurements. At the moderately fast tip scan speed applied in this study $\left(\sim 2 \times 10^{5} \mathrm{pN} / \mathrm{s}\right)$, the thermal drift within the millisecond time scale for one AFM measurement is not a significant factor for the application of the Poisson statistical method. This effect, were it present, would have shown up in the variance vs mean plots. ${ }^{18}$

(13) Lo, Y.-S.; Huefner, N. D.; Chan, W. S.; Stevens, F.; Harris, J. M.; Beebe, T. P., Jr. Langmuir 1999, 15, 1373-1382.

(14) Williams, J. M.; Taejoon, H.; Beebe, T. P., Jr. Langmuir 1996, 12 , $1291-1295$

(15) Han, T.; Williams, J. M.; Beebe, T. P., Jr. Anal. Chim. Acta 1995, $307,365-76$

(16) Wenzler, L. A.; Moyes, G. L.; Harris, J. M.; Beebe, T. P., Jr. Anal. Chem. 1997, 69, 2855-2861.

(17) Wenzler, L. A.; Moyes, G. L.; Raiker, G. N.; Hansen, R. L.; Harris,

J. M.; Beebe, T. P., Jr. Langmuir 1997, 13, 3761-3768.

(18) Stevens, F.; Lo, Y.-S.; Harris, J. M.; Beebe, T. P., Jr. Langmuir 1999, 15, 207-213.

(19) Wei, Z. Q.; Wang, C.; Zhu, C. F.; Zhou, C. Q.; Xu, B.; Bai, C. L. Surf. Sci. 2000, 459, 401-412.

(20) Ibrahim, T. H.; Burk, T. R.; Etzler, F. M.; Neuman, R. D. J. Adhesion Sci. Technol. 2000, 14, 1225-1242.

(21) van der Vegte, E. W.; Hadziioannou, G. Langmuir 1997, 13, 43574368.

(22) Cleveland, J. P.; Manne, S.; Bocek, D.; Hansma, P. K. Rev. Sci. Instrum. 1993, 64, 403-405.

(23) The bond-rupture time $\tau_{\mathrm{R}}$ during an AFM measurement is typically in the range $10^{-2}$ to $10^{-4} \mathrm{~s}$, depending on the rate of force loading applied.

(24) Weber, P. C.; Ohlendorf, D. H.; Wendoloski, J. J.; Salemme, F. R Science 1989, 243, 85-88.

(25) Based on the known crystal structure, the binding between biotin and streptavidin involves five hydrogen bonds, van der Waals interactions, and the ordering of two loops. ${ }^{24}$ Avidin, an analogue to streptavidin, shares $38 \%$ sequence identity and is assumed to exhibit similar bonding with biotin

(26) Miyamoto, S.; Kollman, P. A. Proteins 1993, 16, 226-245.

(27) Chilkoti, A.; Stayton, P. S. J. Am. Chem. Soc. 1995, 117, 1062210628

(28) Although force-induced unbinding processes of ligand-receptor pairs have been simulated in several illuminating molecular dynamics (MD) studies, ${ }^{7,10,30} \mathrm{MD}$ is still currently limited to events of subpicosecond time duration, making its direct relevance to millisecond experiments such as ours questionable. Even with a significant enhancement in computer speed, it will remain a great challenge to simulate AFM bond-rupture processes occurring on the millisecond time scale with MD. Thus we chose to apply a thermodynamic approach to interpret the temperature dependence of unbinding forces in this study.

(29) During the course of a single experiment, tip-surface bonds loaded early (i.e., at the perimeter of the tip contact area where the tip-surface distance increases to the rupture threshold first) will break first and are not part of the data set measured here. This leaves a small ensemble of a few remaining linkages, all of which are stretched essentially to the same extent. This last population of several remaining linkages is what we probe in our experiments and what we model in our theory. As we have shown in earlier work, ${ }^{18}$ if the last remaining set of linkages were not of equal strength, or were not loaded similarly, this would manifest in the variance vs mean plot (e.g., see Figure 2B) as curvature. This did not occur in our systems, which further supports this assumption.

(30) Balsera, M.; Stepaniants, S.; Izrailev, S.; Oono, Y.; Schulten, K. Biophys. J. 1997, 73, 1281-1287.

(31) There is nothing special about the Morse potential in this analysis, and we could have just as easily chosen another form. For example, if a potential of the form $V=4 D_{\mathrm{e}}\left[(\sigma / r)^{12}-(\sigma / r)^{6}\right]$ is assumed, the critical distance is $r_{\text {crit }}=(26 / 7)^{1 / 6} \sigma$ and $V\left(r_{\text {crit }}\right)=-(133 / 169) D_{\mathrm{e}}=-0.79 D_{\mathrm{e}}$. So, one must deposit only $21 \%$ of $D_{\mathrm{e}}$ into bond stretching energy to effect rupture. Of course, the actual potential is neither of the Morse nor 6-12 form precisely. Nevertheless, these examples illustrate that probably only $20-25 \%$ of the total bond dissociation energy must be deposited before the critical distance is reached.

(32) Swamy, M. J. Biochem. Mol. Biol. Int. 1995, 36, 219-225.

(33) Using $100-200 \mathrm{~cm}^{-1}$ as a characteristic interfragment frequency for hydrogen bond motion and an approximate reduced mass of $17 \mathrm{amu}$ $\left(\mathrm{OH}\right.$ vibration against a heavier protein acceptor group) gives, via $h c \lambda^{-1}=$ $\hbar\left(k_{\text {bond }} / \mu\right)^{1 / 2}$, a $k_{\text {bond }}$ value in this range (i.e., $\left.10-40 \mathrm{~N} \cdot \mathrm{m}^{-1}\right)$, consistent with our findings.

(34) Compared to the value for the covalent bond of $\mathrm{HCl}$ ( $b \sim 1.9 \times$ $\left.10^{10} \mathrm{~m}^{-1}\right),{ }^{35}$ the Morse potential parameter $b$ calculated in our analysis $\left(\sim 6.6 \times 10^{9} \mathrm{~m}^{-1}\right)$ is reasonable for the biotin-avidin complex, a strong noncovalent binding that contains several hydrogen bonds and van der Waals interactions.

(35) Vinogradov, S. N.; Linnell, R. H. Hydrogen bonding; Van Nostrand Reinhold Co.: New York, 1971. 